\title{
An End-to-End Cross-Layer Profiling Study of Congestion Control in High BDP Wireless Networks
}

\author{
Homayoun Yousefi'zadeh Xiaolong Li Amir Habibi \\ Department of EECS \\ University of California, Irvine \\ [hyousefi, xiaolonl, ahabibil] @uci.edu
}

\begin{abstract}
We describe a cross-layer study that attempts at profiling the performance of end-to-end congestion control protocols in high BDP wireless networks. Packets transmitted over such networks are assumed to face temporally correlated random bit errors caused by fading and/or blocking. We utilize a finite-state Markov chain to model bit error characteristics and apply per packet link layer FEC codes in order to compensate for such errors. Utilizing our model along with loss expressions, we profile the end-to-end performance of XCP, VCP, and TCP/AQM+ECN congestion control protocols. Based on our profiling results, we observe that (1) the performance of any congestion control protocol highly degrades without properly protecting its data and signaling information against random bit errors, (2) utilizing multiple antenna nodes improves the transient characteristics of the congestion control protocol in terms of utilization and packet error rates, and (3) XCP and VCP represent the best alternatives of congestion control for high bandwidth moderate delay and moderate bandwidth large delay environments, respectively.
\end{abstract}

Index Terms-Wireless Networks, LOS Links, Markov chain, TCP, AQM, ECN, XCP, VCP.

\section{INTRODUCTION}

The Additive-Increase Multiplicative-Decrease (AIMD) property of TCP allows for offering both efficiency and fairness of congestion control. However, utilizing AIMD results in performance degradation of TCP due to the slow start phenomenon. In order to address some of the limitations of TCP, the use of end-to-end congestion notification feedback schemes such as TCP+AQM/ECN has been proposed [10], [4], [5], [15], [18], [13]. While the use of TCP+AQM/ECN can result in reducing loss rate and queue size, it is still not capable of achieving high utilization in high Bandwidth-Delay Product (BDP) networks [16], [11], [17].

eXplicit Congestion-control Protocol (XCP) [14] and Variable-structure Congestion-control Protocol (VCP) [20] are a pair of recently proposed end-to-end protocols that attempt at addressing the problems of TCP. Both protocols require the participation of intermediary nodes to set congestion related information in the header of packets as they travel form their sources to their destinations. A destination transmits acknowledgment packets to its source in order to notify it of congestion phenomena thereby adjusting its transmission rate. Unlike TCP, $\mathrm{XCP}$ and VCP decouple the efficiency of congestion control from its fairness allowing for employing different strategies to cope with efficiency and fairness. The premise of XCP is to

This work was sponsored by the grants from Boeing Integrated Defense Systems and UC Discovery grant number com05-10196 Industry-University Cooperative Research Program. use a Multiplicative-Increase Multiplicative-Decrease (MIMD) scheme for efficiency and an AIMD scheme for fairness. The design goal of VCP is to provide a TCP-like scheme for wired networks. VCP creates a three-region regime of operation referred to as Multiplicative-Increase Additive-Increase Multiplicative-Decrease (MIAIMD). The MI region is utilized to eliminate the slow start characteristic of TCP while the AI and MD regions preserve the fairness characteristics of TCP. For both protocols, the decoupling can result in improving the efficiency of congestion control measured in terms of smaller queues, fewer drops, offering flexible fairness schemes, and providing a better scalability by virtue of not carrying any perflow state information.

A comparison of XCP and VCP reveals the following facts. $\mathrm{XCP}$ requires the use of multiple bits in the IP header of each packet to relay congestion information. While an implementation of XCP in the Linux kernel uses 16 bits [24], the current IETF draft for XCP [9] proposes the use of 128 bits in the IP header. On the contrary, VCP proposes encoding congestion information into two ECN bits. Since the amount of information relayed in the IP header in the case of VCP is much smaller than the case of XCP, VCP represents a more practical protocol alternative than XCP in terms of implementation. However, the better practicality characteristic of VCP may come at the expense of a higher convergence time than that of XCP. That said, the latter depends on the bandwidth and delay characteristics of the traffic as we will show in our reported results.

Aside from the issues related to wired networks, the behavior of any congestion control protocol in wireless networks is affected by the unique characteristics of those networks. One such characteristic is that transmission over wireless links is subject to random bit errors in addition to packet erasures. Hence, for a packet transmitted over wireless links there are three possibilities indicating the packet is (1) delivered error free, (2) delivered corrupted due to random bit errors, or (3) lost due to congestion. We note that the second possibility is usually ignored in wired networks. On the contrary, the performance of any protocol utilizing IP header bits to carry congestion information may be seriously crippled in wireless networks without proper compensation against random bit errors. In [7] and [26], the performance of TCP Reno and Tahoe over wireless links characterized by rich scattering and Rayleigh fading is examined. In [23], the performance of VCP is compared to that of $\mathrm{TCP} / \mathrm{AQM}+\mathrm{ECN}$ in low BDP rich scattering wireless networks.

This paper proposes a cross-layer framework to study the performance of XCP, VCP, and TCP/AQM+ECN in high BDP 
wireless networks. It differentiates from the prior art work in the sense that (1) it provides a more detailed analysis related to Markov channel modeling of the wireless channel as it pertains to the number of antennas, modulation, channel coding; (2) it develops a loss model matching the observed loss pattern of wireless links; and (3) it examines and compares the performance of XCP, VCP, and TCP/AQM+ECN as end-to-end congestion control alternatives in high BDP wireless networks utilizing Line Of Sight (LOS) and Satellite Communications (SATCOM) links.

The rest of this paper is organized as follows. Section II describes the characteristics of the physical and data link layers utilized in our study. It explains how a wireless channel can be modeled by a finite-state Markov chain the parameters of which are related to the number of transmit/receive antennas and modulation. The section also explains how the use of Forward Error Correction (FEC) schemes at the link layer can combat the fading/blockage effects. Section III contains the results of our experiments and the related discussion. Finally, Section IV concludes the paper.

\section{PHYSICAL AND LINK LAYER ANALYSIS}

In this section we analyze the underlying physical and link layers of the wireless channel and provide hooks for relating the effects of such layers to the performance of networks and transport layers where the congestion control protocols are hosted.

Our discussion covers the modeling of fading channel with finite-state Markov chains and the channel coding scheme proposed for compensating the effects of correlated loss. Our modeling approach of fading channels is general and can be applied to both Rayleigh and Rician fading channels representing rich scattering and LOS transmission environments.

\section{A. Markov Chain Modeling of Wireless Links}

As pointed out in [21], [25], [22], and many other articles, a Multiple-Input Multiple-Output (MIMO) fading channel is characterized by temporally correlated loss. The two-state Gilbert-Elliott (GE) loss model [8] provides an elegant mathematical model to capture the loss behavior of such channel. In the GE model, the loss pattern of a bitstream transmitted over a MIMO fading channel is described by a two-state Markov chain. The GOOD state introduces a probability $\gamma$ of staying in the GOOD state and a probability $1-\gamma$ of transitioning to the BAD state while the BAD state introduces a probability $\beta$ of staying in the BAD state and a probability $1-\beta$ of transitioning to the GOOD state. The parameters $\gamma$ and $\beta$ can be measured from the observed average burst lengths of the wireless channel [12].

The GOOD state also represents bit loss with a probability $\varepsilon_{G}$ while the BAD state represents bit loss with a probability $\varepsilon_{B}$ where $\varepsilon_{G}<<\varepsilon_{B}$. The pair of probabilities $\varepsilon_{G}$ and $\varepsilon_{B}$ can be calculated from the statistics of the fading channel, transmission powers, average noise power, shadowing, modulation, number of transmit/receive antennas, and even in the presence of co-channel interference. As such, $\varepsilon_{G}$ and $\varepsilon_{B}$ can also be related to quantities such as signal-to-noise ratio or signal-to-interference-noise ratio. For example, the GOOD and
BAD states can be differentiated by $S N R_{G}$ and $S N R_{B}$ where $S N R_{G}>S N R_{B}$. Since for a LOS channel the two states are typically associated with non-blocked and blocked channels, the quantity $\varepsilon_{G}$ associated with a non-blocked LOS channel is sometimes set to zero.

Let $\varphi(t, r, G)$ and $\varphi(t, r, B)$ denote the probability of receiving $r$ bits from $t$ transmitted bits and winding up in the GOOD and the BAD state of the Gilbert-Elliott model, respectively. Then the overall probability of receiving $r$ bits from $t$ transmitted bits for the Gilbert-Elliott model is given by

$$
\varphi(t, r)=\varphi(t, r, G)+\varphi(t, r, B)
$$

where the recursive probabilities $\varphi(t, r, G)$ and $\varphi(t, r, B)$ are given [25] by

$$
\begin{aligned}
& \varphi(t, r, G)= \\
& \varepsilon_{G}[\gamma \varphi(t-1, r, G)+(1-\beta) \varphi(t-1, r, B)] \\
& \left(1-\varepsilon_{G}\right)[\gamma \varphi(t-1, r-1, G) \\
& \quad+(1-\beta) \varphi(t-1, r-1, B)]
\end{aligned}
$$

and

$$
\begin{aligned}
& \varphi(t, r, B)= \\
& \varepsilon_{B}[(1-\gamma) \varphi(t-1, r, G)+\beta \varphi(t-1, r, B)] \\
& \quad\left(1-\varepsilon_{B}\right)[(1-\gamma) \varphi(t-1, r-1, G) \\
& \quad+\beta \varphi(t-1, r-1, B)]
\end{aligned}
$$

for $t \geq r>0$ and the initial conditions

$$
\begin{aligned}
\varphi(0,0, G) & =g_{s s}=\frac{1-\beta}{2-\gamma-\beta} \\
\varphi(0,0, B) & =b_{s s}=\frac{1-\gamma}{2-\gamma-\beta} \\
\varphi(1,0, G) & =\varepsilon_{G}\left[\gamma g_{s s}+(1-\beta) b_{s s}\right] \\
\varphi(1,0, B) & =\varepsilon_{B}\left[(1-\gamma) g_{s s}+\beta b_{s s}\right]
\end{aligned}
$$

The quantities $\varepsilon_{G}$ and $\varepsilon_{B}$ can be calculated in terms of modulation, number of transmit/receive antennas, and average received signal-to-noise ratios. Further, the average values of signal-to-noise and signal-to-interference-noise ratios can be extracted from the fading channel statistics, transmission powers, average noise power, and shadowing even in the presence of co-channel interference. The details of calculations for rich scattering links characterized by Rayleigh fading and LOS links characterized by Rician fading are discussed in [25] and [22], respectively.

In [21], closed-form expressions describing the modulation symbol error rate of MIMO wireless links are identified in terms of the number of signal points in the constellation $M$ and the average received signal-to-noise ratio. The calculations are carried out under the assumption of facing a flat fading Rayleigh channel and utilizing Phased Shift Keying (PSK) modulation. In what follows we apply the previous results to capture the modulation symbol error rates of both rich scattering and LOS wireless links which may be accommodating multiple antenna nodes.

First, we introduce the modulation symbol error rate of a link associated with single transmit and $N$ receive antenna nodes using Maximum Ratio Combining (MRC) as

$$
\begin{aligned}
& \varepsilon=\frac{M-1}{M}-\frac{1}{\pi} \sqrt{\frac{\vartheta}{1+\vartheta}}\left\{\left(\frac{\pi}{2}+\tan ^{-1} \xi\right) \sum_{j=0}^{N-1}\left(\begin{array}{c}
2 j \\
j
\end{array}\right) \frac{1}{[4(1+\vartheta)]^{j}}\right. \\
& \left.+\sin \left(\tan ^{-1} \xi\right) \sum_{j=1}^{N-1} \sum_{i=1}^{j} \frac{\sigma_{i j}}{(1+\vartheta)^{j}}\left[\cos \left(\tan ^{-1} \xi\right)\right]^{2(j-i)+1}\right\}
\end{aligned}
$$


where $\vartheta=S N R \sin ^{2}\left(\frac{\pi}{M}\right), \xi=\sqrt{\frac{\vartheta}{1+\vartheta}} \cot \frac{\pi}{M}$, and $\sigma_{i j}=$ $\frac{\left(\begin{array}{c}2 j \\ j\end{array}\right)}{\left(\begin{array}{c}2(j-i) \\ j-i\end{array}\right) 4^{i}[2(j-i)+1]}$. From Equation (5), one can calculate the modulation symbol error rate of a link associated with single transmit single receive antenna nodes as well as a link associated with single transmit double receive antenna nodes by setting $N$ to 1 and 2 , respectively. Relying on a discussion of diversity gains, we also argue that the modulation symbol error rate of Space-Time Block Codes (STBCs) of [3] and [19] can be calculated from Equation (5) by proper mapping of the values of $S N R$. For example, the modulation symbol error rate of a link associated with double transmit single receive antenna nodes can be calculated by replacing $S N R$ with $\frac{S N R}{2}$ and setting $N$ to 2 in Equation (5). Similarly, the modulation symbol error rate of a link associated with double transmit double receive antenna nodes can be calculated by replacing $S N R$ with $\frac{S N R}{2}$ and setting $N$ to 4 in Equation (5).

We note that a modulation symbol is mapped to a bit for BPSK. Consequently, it suffices to replace $S N R$ with $S N R_{G}$ and $S N R_{B}$ in Equation (5), in order to calculate modulation symbol error rate $\varepsilon_{G}$ of the GOOD state and $\varepsilon_{B}$ of the BAD state of the GE loss model. We note that while our choice of BPSK reflects a matter of convenience for our study, it does not affect the generality of our model.

\section{B. Channel Coding and Associated Error Modeling}

As indicated by various standards such as Direct Sequence Multiple Access Code Division (DS-CDMA) [2], the use of Forward Error Correction schemes at the link layer has been proposed in order to combat the fading effects of wireless channels described above.

In our work, we propose the use of Reed-Solomon (RS) channel coders at the link layer as an optional component to compensate for temporally correlated loss effects of the wireless channel. We note that an RS channel coder $R S(b, k)$ converts $k$ channel coding symbols into a $b$-symbol block by appending $(b-k)$ parity symbols. Such channel coder is able to correct as many as $t_{C}=\left\lfloor\frac{b-k}{2}\right\rfloor$ symbol errors in a block.

We distinguish between the modulation symbols and channel coding symbols by noting that a channel coding symbol typically consists of a number of modulation symbols. For example, an 8-bit RS channel coding symbol consists of eight BPSK modulation symbols. If at least $b-t_{C}$ channel coding symbols are correctly received from $b$ transmitted channel coding symbols, the whole block is recoverable. We note that our approach calls for mapping a block onto a packet. Since an RS channel coder $R S(b, k)$ at the link layer treats an assembled packet as its data block $k$, errors in both the payload and headers/trailers of the network and higher layers can be mitigated for each packet individually at the receiving side.

Next, we discuss our error modeling approach. Suppose the RS coder generates a set of channel coding symbols where each symbol consists of $s$ bits. A channel coding symbol is received error free if all of its $s$ bits are received free of errors. As indicated earlier, we assume that the loss pattern of the wireless channel at the bit level is described by the GE model. Thus, the probability of receiving a channel coding symbol free of error under the GE model is obtained from Equation (1) with $t=r=s$ as $\varphi(s, s)$.

Since the inter-symbol temporal correlation is captured in the expression $\varphi(s, s)$ for back-to-back transmitted channel coding symbols, we can obtain the probability of channel coding symbol block loss as

$$
\Psi\left(b, t_{C}, \varepsilon_{G}, \varepsilon_{B}\right)=\sum_{i=0}^{b-t_{C}-1}\left(\begin{array}{l}
b \\
i
\end{array}\right)(1-\varphi(s, s))^{i} \varphi(s, s)^{(b-i)}
$$

In order to validate the results of our channel modeling in the presence of RS coding, we observe the loss behavior of a pointto-point link utilized for transmitting a packetized bitstream. We, then, compare the observed loss rate with the calculated loss rate of Equation (6).

Fig. 1 provides sample validation results of our loss model. For a channel coding symbol size of eight bits, BPSK modulation, a single transmit single receive $(1 \times 1)$ antenna configuration, and for three different choices of transmitted byte lengths $L$, the figure compares the calculated average byte loss from our model with the observed average byte loss over a range of values of $S N R_{G}$ in the GE model with $S N R_{G}=10 S N R_{B}$. The other parameters of the GE model are set to $\gamma=0.99875$ and $\beta=0.875$. The averages are calculated over one million experiments. As observed from the plots, the values of calculated and observed average byte loss match in all three cases. We have observed that while the choices of GE model parameters, modulation, and channel coding symbol sizes affect our results, the validation results remain the same.

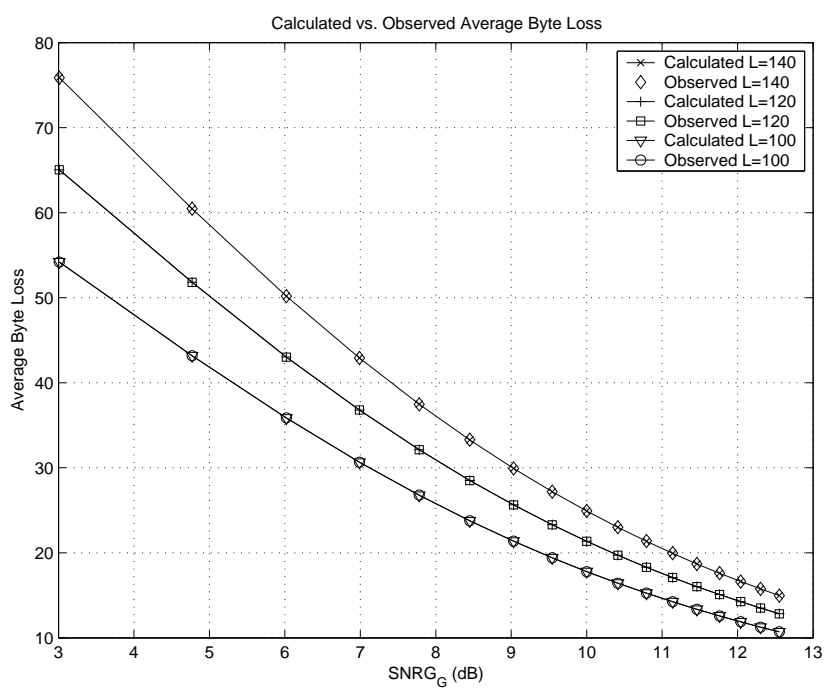

Fig. 1. A comparison of calculated and observed average byte loss for a $1 \times 1$ antenna configuration, a channel coding symbol size of eight bits, and BPSK modulation.

\section{Profiling of Performance}

In this section, we describe performance profiling results of the congestion control protocols of our study over wireless networks formed by MIMO links.

We experiment with a variety of parameter settings. While the trends of reported results are general, the followings represent the various settings of the wireless channel and physical 
layer for the reported results. The transition probabilities of the GE model are set as $\gamma=0.99875$ and $\beta=0.875$ representing average burst lengths of 800 and 8 bits for the GOOD and BAD state, respectively. For the same $\gamma$, we also experiment with $\beta=0.91667$ representing an average burst length of 12 bits. Further, we set $S N R_{G}=10 S N R_{B}$ to differentiate between the qualities of the link in the GOOD and BAD states. Four different combinations of antennas are considered as (1) single transmit single receive $(1 \times 1)$; $(2)$ double transmit single receive $(2 \times 1)$; $(3)$ single transmit double receive $(1 \times 2)$; and (4) double transmit double receive $(2 \times 2)$ antennas. We utilize BPSK modulation along with a channel coding symbol size of one byte when using RS coding FEC. We note that the channel coding rate is defined as $r=k / b$ when using FEC.

The illustrations of Fig. 2 show the dumbbell topology of our experiments with either a direct wireless bottleneck link or a pair of SATCOM bottleneck links. Each platform includes a number of nodes wired to a multiple antenna radio router playing the role of the gateway for its platform. We assume that the direct wireless link is identified by bandwidth and delay characteristics of $54 \mathrm{Mbps}$ and $80 \mathrm{msec}$, respectively. The SATCOM links are assumed to have bandwidth and delay characteristics of $2 \mathrm{Mbps}$ and $0.5 \mathrm{sec}$, respectively. The capacity of a wired link is assumed to always be twice as high as the capacity of the bottleneck link with a delay of $8 \mathrm{msec}$.

We set the parameters of VCP according to what is reported in Table 1 of [20]. In the case of TCP/AQM+ECN, we utilize TCP Reno along with Random Early Marking (REM) [5]. Particularly, we set the parameters of REM according to what is suggested by [6] at the two ends of the bottleneck link. All of the simulations are repeated 10 times and averaged with individual runs over a total simulation period of up to 1000 seconds.

We select $10 \mathrm{ftp}$ sources in Platform A sending packets to destinations in Platform B and 10 ftp sources in Platform B sending packets to destinations in Platform A. When active, each source can generate packets at the rate of its dedicated link. Each data packet has a size of 1040 bytes and each acknowledgment packet has a size of 40 bytes. All of the links operate in a full-duplex mode and link bandwidths are shared among data and acknowledgment packets. In the duration of an individual run, each flow starts as soon as its session is established and continues until either its session is torn down due to fading-, blockage-, and congestion-related loss or the simulation experiment has come to an end. As such, each flow follows a random start time with a random duration. We do not collect the statistics in the first 10 seconds of each run to allow for stabilizing the transitioning behavior of NS2. While we only report a small number of our results due to the shortage of space, we note that our findings are fairly consistent across a wide range of experiments.

Fig. 3 compares the performance of the three protocols for $\gamma=0.99875, \beta=0.91667$, a channel coding rate of $r=0.9$, and different choices of antenna configurations. It plots the total number of delivered packets versus $S N R_{G}$ for the direct wireless link scenario. The interesting observations are that (1) the use of different antenna configurations affects the transient behavior in the case of each protocol but not the steady-state behavior and (2) the steady-state performance of XCP and VCP

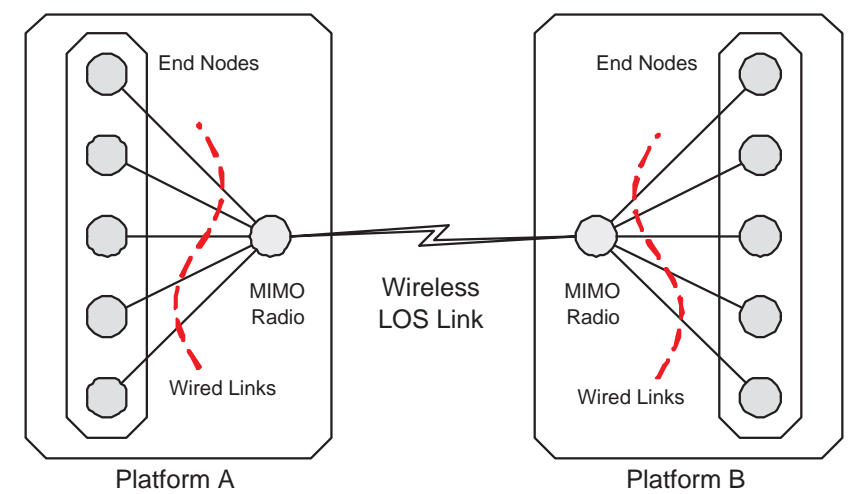

(a)

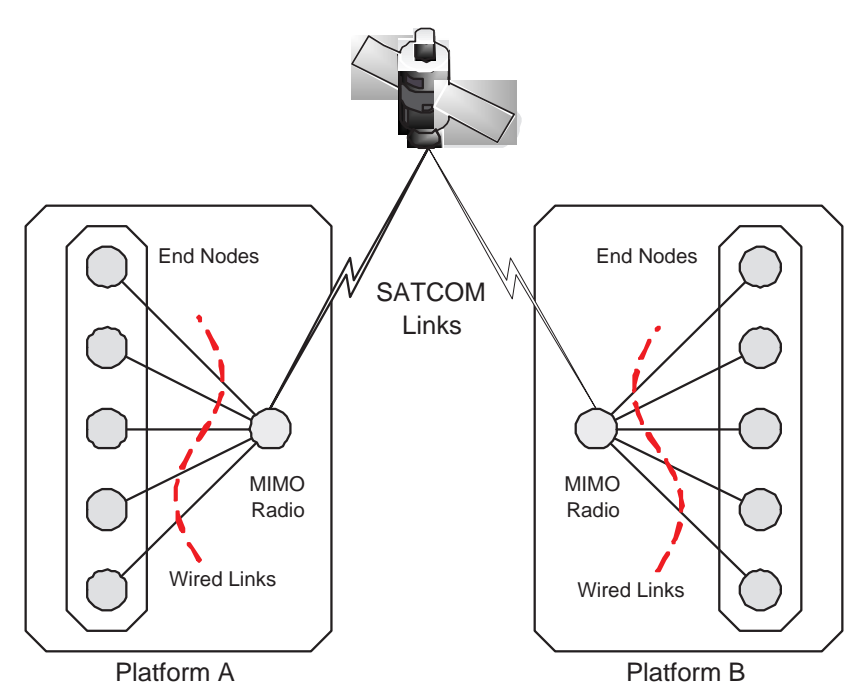

(b)

Fig. 2. An illustration of the communication topologies used in our experiments utilizing: (a) a direct wireless link and (b) a pair of SATCOM links.

is much better than that of TCP/AQM+ECN. The first observation is justified as both $S N R_{G}$ and the antenna configurations affect the quality of the link. Each protocol exhibits its steadystate behavior once the quality of the link is improved. When comparing the results of different antenna configurations, we observe that the performance of $1 \times 1,2 \times 1,1 \times 2$, and $2 \times 2$ are in an ascending order as the result of improving SER from the former to the latter configuration. The second observation shows that both XCP and VCP perform much better in high BDP environments with XCP taking the lead when the increase in BDP product is mostly due to bandwidth rather than delay.

Fig. 4 examines the effects of applying different FEC rates to data packets of VCP for $1 \times 1$ and $2 \times 2$ antenna configurations. Fig. 5 measures similar effects in the cases of VCP and $\mathrm{XCP}$ for a $1 \times 2$ antenna configuration. In all cases, the FEC rate of acknowledgment packets is set to 0.5. It is observed from the figures that introducing a small percentage of FEC to the data packets and a more significant percentage of FEC to acknowledgment packets at the link layer can significantly improve the performance of both VCP and XCP. The latter is due to the fact that VCP and XCP sources collect information about the status of the network from the acknowledgment packets. Since acknowledgment packets are only 40 bytes long and fad- 


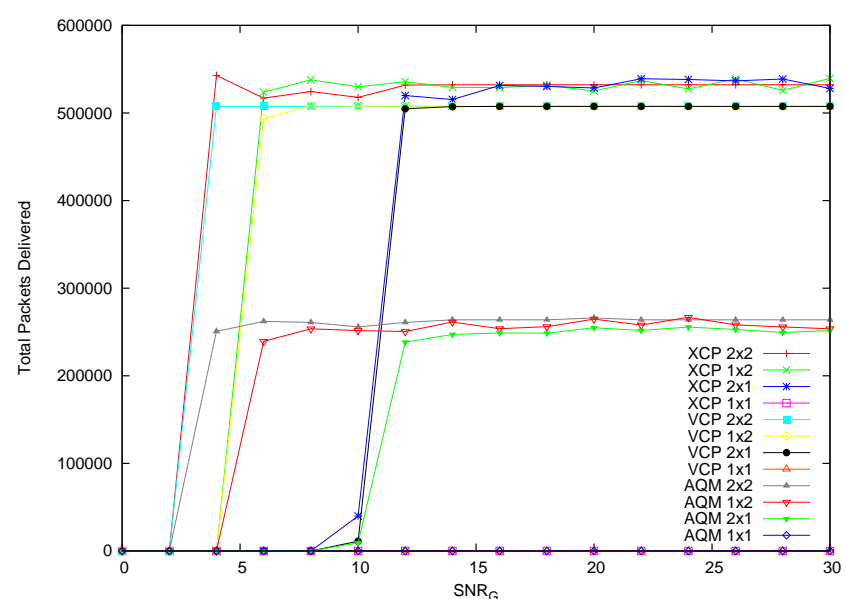

Fig. 3. A performance comparison of $\mathrm{XCP}, \mathrm{VCP}$, and $\mathrm{TCP} / \mathrm{AQM}+\mathrm{ECN}$ with different antenna configurations of the direct wireless link. The average burst lengths of the GOOD and BAD state are set at 800 and 12 bits. FEC rates of 0.9 and 0.5 are applied to data and acknowledgment packets, respectively.

ing related random errors happen in bursts, they can corrupt the shorter acknowledgment packets much easier than longer data packets. We note that the FEC strength must be chosen with the consideration of antenna configuration, link quality, and protocols. It is our opinion based on experiments that providing stronger protection for acknowledgment packets as opposed to data packets can properly address the tradeoff between protocol efficiency and bandwidth overhead. It is also important to note that sensitivity of XCP, VCP, and TCP/AQM+ECN to the loss of acknowledgment packets is in the descending order.

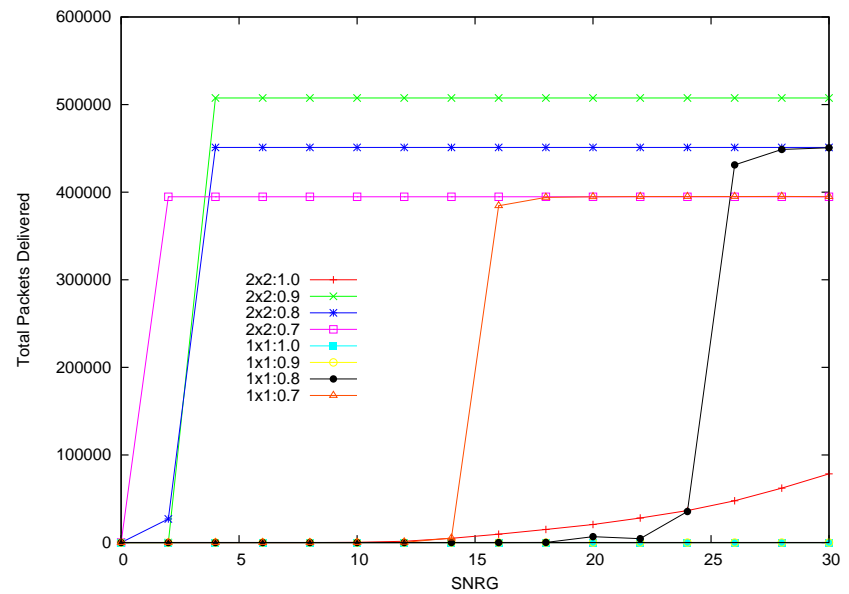

Fig. 4. A comparison of VCP performance for different data FEC rates over a direst wireless link. RS codes with rates $1,0.9,0.8$, and 0.7 are applied over $1 \times 1$ and $2 \times 2$ antenna configuration of the direct wireless link. The average burst lengths of the GOOD and BAD state are set at 800 and 8 bits.

Next, we compare the performance of the three protocols when the channel is identified by $\gamma=0.99875$ and $\beta=0.875$. Fig. 6 sketches Packet Error Rate (PER) versus $S N R_{G}$ for the three protocols and with different choices of antenna configurations. The results are fairly consistent with those shown in Fig. 3.

Up until this point, we have reported our evaluation results over direct wireless links. Next, we focus on the performance

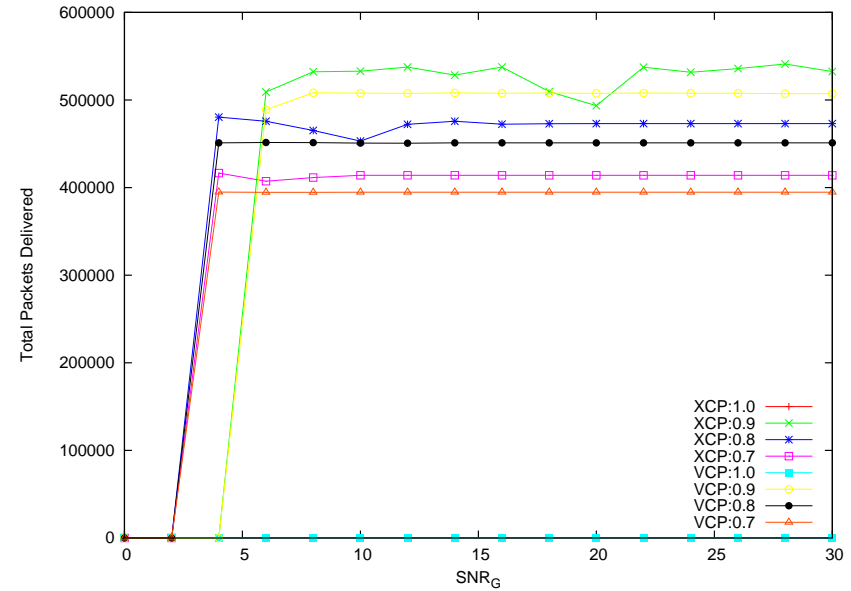

Fig. 5. A comparison of VCP and XCP performance for different data FEC rates over a direst wireless link. RS codes with rates $1,0.9,0.8$, and 0.7 are applied over a $1 \times 2$ antenna configuration of the direct wireless link. The average burst lengths of the GOOD and BAD state are set at 800 and 12 bits.

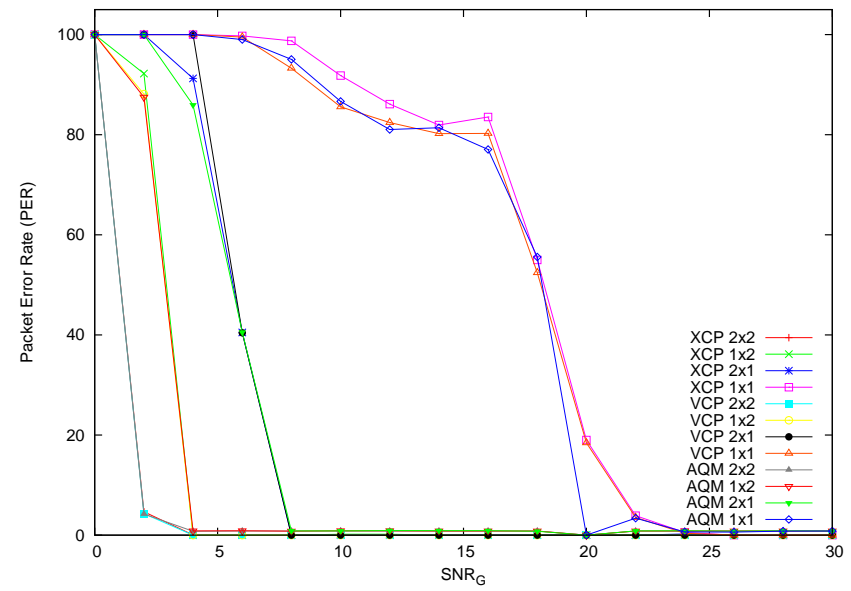

Fig. 6. A performance comparison of XCP, VCP, and TCP/AQM+ECN with different antenna configurations of the direct wireless link. The average burst lengths of the GOOD and BAD state are set at 800 and 8 bits. FEC rates of 0.8 and 0.5 are applied to data and acknowledgment packets, respectively.

evaluation over SATCOM links. We note that the main difference of a SATCOM scenario with a direct wireless link scenario is that the increase in the value of BDP in a SATCOM scenario is mostly due to an increased delay rather than bandwidth. Since the behavior of the protocols remains in part and qualitatively the same as what we have reported so far, we only focus on the major differences. Fig. 7 reports the percentage utilization of the SATCOM links versus the simulation time of 1000 seconds. The data traffic is one way from Platform A to Platform B. The very interesting observation is that VCP outperforms the other two protocols. It converges rapidly and remains at a high utilization without much oscillation. To the contrary, XCP is not as well-behaved in this scenario. While XCP's oscillatory behavior is still better than that of TCP/AQM+ECN, it shows wide variations in utilization. We relate the behavior of the protocols to delay caused timeouts. While the oscillatory behavior of TCP/AQM+ECN relates to TCP's slow start, VCP quickly reacts to timeouts because of utilizing $\mathrm{MI}$ in the low load region. Since XCP does not utilize the same design in the 
low load region the way VCP does, it takes longer for XCP to react to timeouts. It is also important to note that the results of two way traffic when each platform transmits to the other platform, is the exacerbated version of the one way traffic case for both XCP and TCP/AQM+ECN. However, VCP still shows high utilization with very little oscillations.

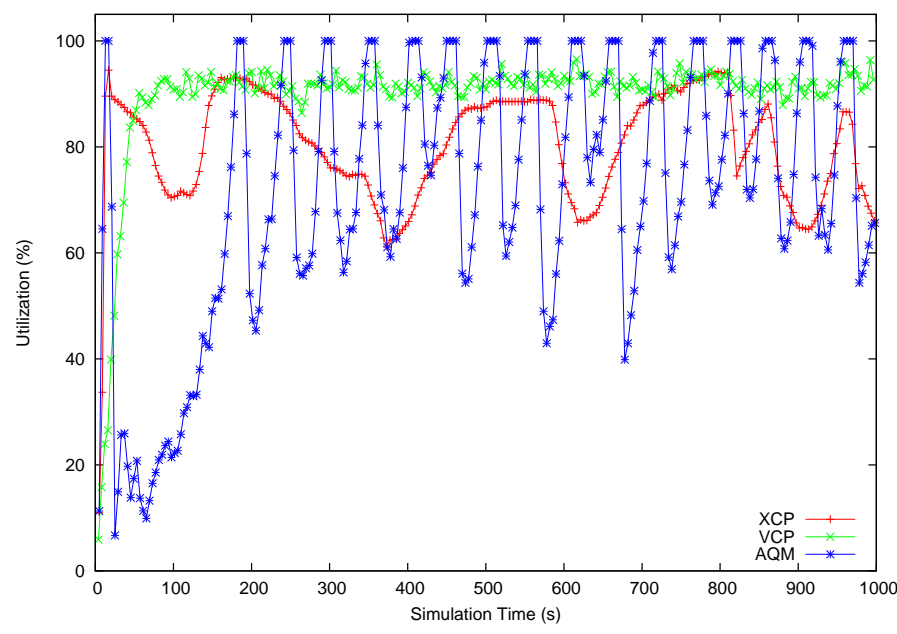

Fig. 7. A performance comparison of XCP, VCP, and TCP/AQM+ECN with $2 \times 2$ antenna configuration, $S N R_{G}=30 \mathrm{~dB}$, and over a pair of SATCOM links. The average burst lengths of the GOOD and BAD state are set at 800 and 8 bits. FEC rates of 0.93 and 0.5 are applied to data and acknowledgment packets, respectively.

At the end of this section, it is insightful to discuss the effects of a number of parameters. First, we note that the use of larger data packet sizes typically results in requiring to use higher FEC strengths. This is anticipated as the use of larger packet sizes means a larger collection of channel coding symbols to form a block of data. Second, we note that the use of larger channel coding symbol sizes also results in requiring to use higher FEC strengths due to the fact that a channel burst loss is more likely to corrupt at least one bit of the symbol. However, the above issues are traded off against segmentation/reassembly and the decoding complexity, respectively. Finally, we would like to discuss the impacts of having to deal with short-lived flows as oppose to long-lived flows. We have made consistent observations as what is reported by [20] in the case of VCP over wired scenarios, i.e., our results have qualitatively remained the same and the implications have mostly appeared in the form of varying average buffer sizes.

\section{CONCLUSION}

In this paper, we described a cross-layer study focusing on performance profiling TCP/AQM+ECN, XCP, and VCP in high BDP wireless networks. We assumed that the wireless MIMO links introduce random bit errors related to fading and/or blocking. We utilized finite-state Markov chains to model the loss characteristics of the MIMO links and proposed the use of Reed-Solomon FEC codes at the link layer in order to combat the effects of random bit errors. Our performance profiling results illustrated the sensitivity of both XCP and VCP to the loss of congestion information carried in the acknowledgment packets. The results also showed that a moderate use of the link layer FEC codes could help compensate for such effects. Given that acknowledgment packets are reasonably protected by link layer FEC, our results revealed that XCP outperforms VCP in high bandwidth moderate delay scenarios. However, in moderate bandwidth high delay scenarios that are typical of STACOM links, VCP outperformed XCP. Both VCP and XCP outperformed TCP/AQM+ECN in high BDP scenarios of our study.

\section{REFERENCES}

[1] -, "Network Simulator NS2," Available at http://www.isi.edu/nsnam/ns/.

[2] -, "TIA/EIA/IS-99, Data Services Option Standard for Wideband Spread Spectrum Digital Cellular System," 1995. Available at http://electronics.ihs.com/document/abstract/EZGWCAAAAAAAAAAA

[3] S.M. Alamouti, "A Simple Transmitter Diversity Scheme for Wireless Communications," IEEE JSAC, November 1998.

[4] M. Allman, V. Paxson, W. Stevens, "TCP Congestion Control," IETF RFC 2581, April 1999.

[5] S. Athuraliya, V. Li, S. Low, and Q. Yin, "REM: Active Queue Management," IEEE Network, May 2001.

[6] S. Athuraliya, "A Note on Parameter Values of REM with Reno-like Algorithms," Available at . http://netlab.caltech.edu/pub/papers/REMparameter.pdf, March 2002.

[7] A. Chockalingam, M. Zorzi, R.R. Rao, "Performance of TCP on Wireless Fading Links with Memory,” In Proc. IEEE ICC, 1998.

[8] E.O. Elliott, "Estimates on Error Rates for Codes on Burst-Noise Channels," Bell Syst. Tech. J., September 1963.

[9] A. Falk, D. Katabi, Y. Pryadkin, "Specification for the Explicit Control Protocol (XCP)," IETF Internet Draft, Available at http://www.isi.edu/isixcp/docs/draft-falk-xcp-spec-01.txt, October 2005.

[10] S. Floyd, V. Jacobson, "Random Early Detection Gateways for Congestion Avoidance," IEEE/ACM Trans. Networking, August 1993.

[11] C. Hollot, V. Misra, D. Towsley, W. Gong, "Analysis and Design of Controllers for AQM Routers Supporting TCP Flows," IEEE Trans. Automatic Control, June 2002.

[12] H. Jafarkhani, P. Ligdas, N. Farvardin, "Adaptive Rate Allocation in a Joint Source-Channel Coding Framework for Wireless Channels," In Proc. IEEE VTC, 1996.

[13] C. Jin, D. Wei, S. Low, "FAST TCP: Motivation, Architecture, Algorithms, Performance," In Proc. IEEE INFOCOM, 2004.

[14] D. Katabi, M. Handley, C. Rohrs, "Congestion Control for High Bandwidth-Delay Product Networks," In Proc. of ACM SIGCOMM, 2002.

[15] S. Kunniyur, R. Srikant, "Analysis and Design of an Adaptive Virtual Queue (AVQ) Algorithm for Active Queue Management," In Proc. ACM SIGCOMM, 2001.

[16] T. Lakshman, U. Madhow, "The Performance of TCP/IP for Networks with High Bandwidth-Delay Products and Random Loss," IEEE/ACM Trans. Networking, June 1997.

[17] S. Low, F. Paganini, J. Wang, and J. Doyle, "Linear Stability of TCP/RED and a Scalable Control," Computer Networks Journal, December 2003.

[18] K. K. Ramakrishnan, S. Floyd, "The Addition of Explicit Congestion Notification (ECN) to IP," IETF RFC 3168, September 2001.

[19] V. Tarokh, H. Jafarkhani, A.R. Calderbank, "Space-Time Block Coding from Orthogonal Designs," IEEE Trans. Information Theory, July 1999.

[20] Y. Xia, L. Subramanian, I. Stoica, S. Kalyanaraman, "One More Bit Is Enough," In Proc. ACM SIGCOMM, 2005.

[21] H. Yousefi'zadeh, H. Jafarkhani, M. Moshfeghi, "Power Optimization of Wireless Media Systems with Space-Time Block Codes," IEEE Trans. Image Processing, July 2004.

[22] H. Yousefi'zadeh, "A Constrained Resource Allocation Study for LOS MIMO Fading Ad-Hoc Networks," In Proc. IEEE WCNC, 2006.

[23] H. Yousefi'zadeh, A. Habibi, "A Performance Comparison Study of Endto-End Congestion Control Protocols over MIMO Fading Channels," In Proc. IEEE MILCOM, 2006.

[24] Y. Zhang, T.R. Henderson, "An Implementation and Experimental Study of the eXplicit Control Protocol (XCP)," In Proc. IEEE INFOCOM, 2005.

[25] L. Zheng, H. Yousefi'zadeh, H. Jafarkhani, "Resource Allocation in Fading Wireless Ad-Hoc Networks with Temporally Correlated Loss," In Proc. of IEEE WCNC, 2004.

[26] M. Zorzi, A. Chockalingam, R.R. Rao, "Throughput Analysis of TCP on Channels with Memory,” IEEE JSAC, July 2000. 